\title{
Effect of retained beta layer on slip transmission in Ti-6Al-2Zr-1Mo-1V near alpha titanium alloy during tensile deformation at room temperature
}

\author{
Dong $\mathrm{He}^{\mathrm{a}, *}$, Jingchuan Zhu ${ }^{\mathrm{b}}$, S. Zaefferer ${ }^{\mathrm{c}}$, D. Raabe ${ }^{\mathrm{c}}$ \\ ${ }^{a}$ State Key Laboratory of Nonlinear Mechanics, Institute of Mechanics, Chinese Academy of Sciences, Beijing 100190, China \\ ${ }^{\mathrm{b}}$ National Key Laboratory for Precision Hot Processing of Metals, Harbin Institute of Technology, Harbin 150001, China \\ ${ }^{c}$ Max-Planck-Institut für Eisenforschung, Abteilung Mikrostrukturphysik und Umformtechnik, Max-Planck-Strasse 1, 40237 Düsseldorf, Germany
}

\section{A R T I C L E I N F O}

\section{Article history:}

Received 2 September 2013

Accepted 6 December 2013

Available online 15 December 2013

\section{Keywords:}

Retained beta

Slip transmission

Burgers orientation relationship

Tensile deformation

Titanium alloys

\begin{abstract}
A B S T R A C T
Slip is the main plastic deformation mechanism in titanium alloys at room temperature. This is especially so for near alpha titanium alloy like Ti-6Al-2Zr-1Mo-1V, which contains low beta stabilizing and high aluminum (alpha stabilizing) element additions. The effects of retained beta layers on slip transmission across $\alpha / \beta$ interfaces in Ti-6Al-2Zr-1Mo- $1 \mathrm{~V}$ during tensile deformation have been studied in the current work. High resolution scanning electron microscopy (HR-SEM) and electron backscatter diffraction (EBSD) techniques were used to study the deformation microstructure. The results indicate that the full Burgers crystal orientation relationship (OR) between the $\alpha$ and the thin retained $\beta$ phase layers facilitates slip transition but is not the necessary requirement/restriction. Some novel slip trace morphologies that are characterized by deflection and bifurcation (fork-like morphology) are revealed in the retained $\beta$ layers between two abutting $\alpha$ grains. The possible reasons for these different slip transmission patterns are analyzed by EBSD results and a schematic model is proposed.
\end{abstract}

(c) 2013 Elsevier Ltd. All rights reserved.

\section{Introduction}

As a typical near $\alpha$ titanium alloy, Ti-6Al-2Zr-1Mo-1V (TA15) is widely used for aerospace applications due to its high specific strength, excellent thermal stability, low growth rate of fatigue crack and strong corrosion resistance [1-3]. Because of its high aluminum content, slip is the dominant deformation mechanism for TA15 titanium alloy even at room temperature [4-5].

The mechanical properties of $\alpha$ and near $\alpha$ titanium alloys are mainly determined by their microstructures, especially by the size, the morphology and the crystallographic orientations distribution of the $\alpha$ phase [4,6-9]. In addition, slip nature and distribution during deformation processes are also very important [10-12]. The slip system activation and slip transmission phenomena across grain and phase boundaries were reported in the publications [10-11,13-16]. The slip transmission across low-angle $\alpha / \alpha$ grain boundaries of deformed Ti occurs at room temperature with two verified criteria: (I) the angles between slip planes/directions at boundaries should be minimized and (II) the Burgers vector of the residual dislocation should be minimized [14]. In $\alpha / \beta$ titanium alloy, the mechanism of slip transmission in oriented single-colony crystals has been studied by transmission electron microscopy

\footnotetext{
* Corresponding author. Tel./fax: +86 1082543962.

E-mail address: hedong@imech.ac.cn (D. He).
}

(TEM) and analyzed with a simple model based on the accumulation of residual dislocations at the $\alpha / \beta$ interface [13]. However, this research was limited to a single colony and a very local area imposed by the thinning process of TEM foils. The micro gliding and deformation mechanisms in a $\alpha / \beta$ titanium alloy with polycrystals were described by Bridier et al. [10], who observed slip transmission between the two parallel $\alpha$ lamellae. However, the details of the slip transition have not been revealed. In another study, parallel $\alpha$ lamellae with similar orientation were treated as a single $\alpha$ grain to simplify the analysis; however, due to this simplification the $\beta$ layers between the $\alpha$ lamellae were completely neglected [17].

To our knowledge, the effects of the retained $\beta$ layers on slip mode in the $\beta$ forged [18] (forging deformation of the alloy is done above the beta transus) TA15 titanium alloy, which do not have a perfect Burgers OR between the $\alpha$ and retained $\beta$ layers, have not been investigated. Furthermore, the details of slip line morphology during transfer across the retained $\beta$ layers are also not clear. The current work will focus on these problems. The effects of the crystal orientations of $\alpha$ and retained $\beta$ phase on the slip transmission across $\alpha / \beta$ interface will be studied and discussed. Some novel slip trace morphology during the slip transmission will be revealed. Finally, a schematic model based on EBSD observations and the identification of activated slip systems will be proposed to account for those various slip trace patterns. 


\section{Experimental details}

\subsection{Material}

The material used in this study was TA15 titanium alloy containing $6.47 \mathrm{wt} \% \mathrm{Al}, 1.59 \mathrm{wt} \% \mathrm{Zr}, 1.45 \mathrm{wt} \% \mathrm{Mo}, 1.91 \mathrm{wt} \% \mathrm{~V}$, $0.038 \mathrm{wt} \% \mathrm{Fe}$ and $\mathrm{Ti}$ balance. The material was subjected to $\beta$ forging at $1000{ }^{\circ} \mathrm{C}$ and was subsequently homogenized at $600{ }^{\circ} \mathrm{C}$ for $4 \mathrm{~h}$. The microstructure consisted of coarse $\alpha$ phase (hexagonal close-packed, hcp) lamellae and a few retained $\beta$ phase (body-centered cubic, bcc) layers (less than 10 vol.\%), as shown in Fig. 1.

\subsection{Sample manufacturing and specific surface preparation}

The flat samples of $49 \mathrm{~mm}$ in length and $2 \mathrm{~mm}$ in thickness for tensile tests were machined from a wrought bar stock along the elongated direction, as shown in Fig. 2a. The gauge length of the tensile sample is $30 \mathrm{~mm}$ (see Fig. 2a). The flat surfaces of the tensile samples were prepared for SEM observations and EBSD measurements through standard grinding and polishing routines (ASTM: E3-11 (http://www.astm.org/Standards/E3.htm)). The final polishing was performed with a polishing solution consisting of colloidal silica (OP-S, 90 vol.\%) and $\mathrm{H}_{2} \mathrm{O}_{2}$ (10 vol.\%).

\subsection{Tensile tests and EBSD measurements}

Two tensile tests[19], labeled as $A$ and $B$, were performed at the same strain rate $\left(0.7 \times 10^{-4} \mathrm{~s}^{-1}\right)$. Tensile test sample $A$ and $B$ were conducted up to the macroscopic strain of $2.4 \%(0.5 \%$ residual plastic strain, see Fig. 1b) and 4.2\% (2.3\% residual plastic strain, see Fig. 1c), respectively.

The polished surfaces of the samples were carefully protected during the tensile deformation for SEM and EBSD observation. EBSD-based orientation mapping was carried out on a JEOL $6500 \mathrm{~F}$ scanning electron microscope equipped with an EBSD system developed by EDAX/TSL ${ }^{\circledR}$. Since the present investigations are focused on the influence of the retained/thin $\beta$ layers, the EBSD measurements were performed with a fine scanning step size of $0.1 \mu \mathrm{m}$. The morphology of the slip lines and the slip transmission through the retained $\beta$ layers are revealed by high magnification SEM image. The activated slip systems are identified by the combination of HR-EBSD maps. (a)
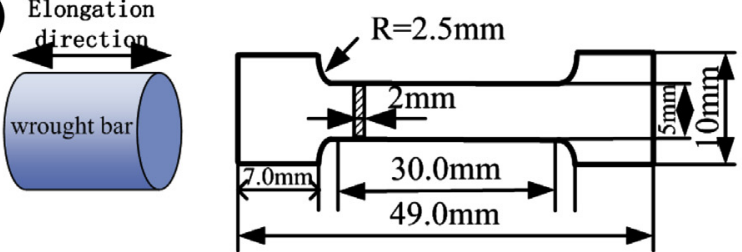

(b)

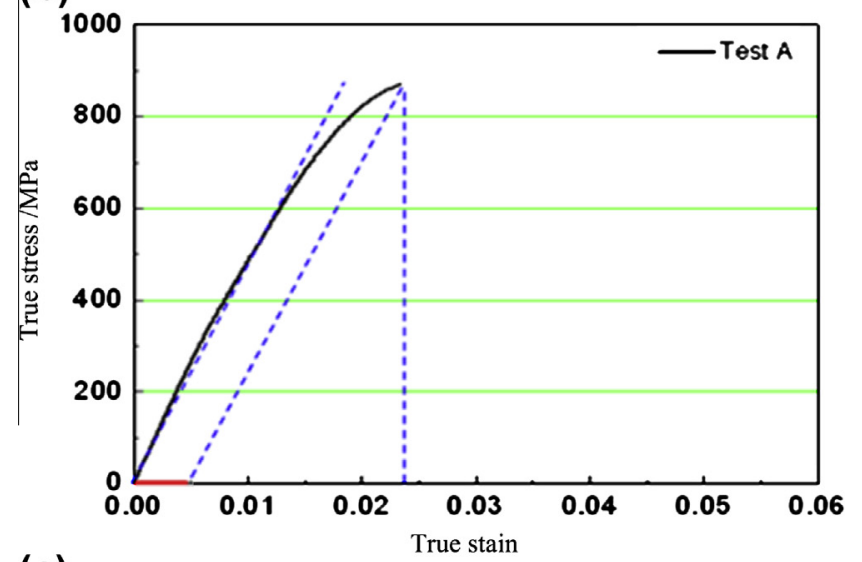

(c)

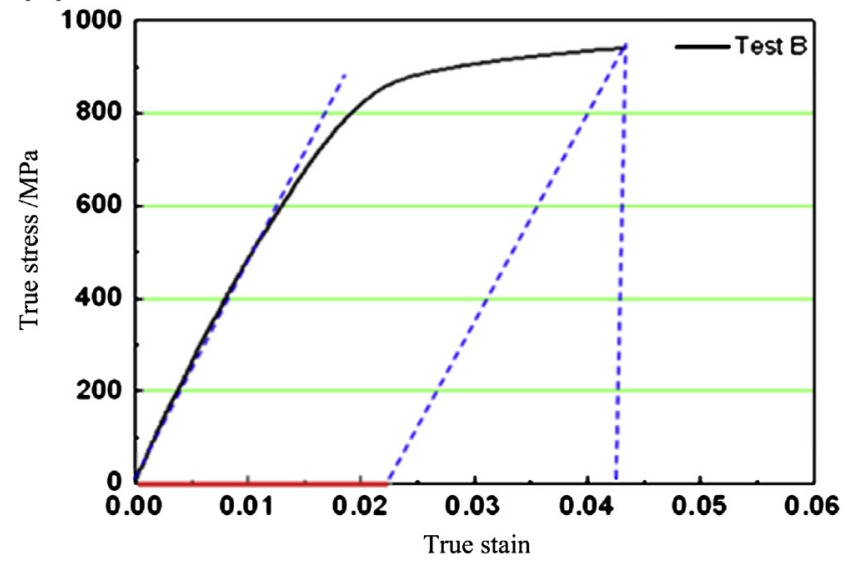

Fig. 2. The sketch map of tensile sample and the true stress-strain curves. (a) The sketch map of tensile sample machining and the geometry of the tensile sample. (b) The true stress-strain curve of sample A. (c) The true stress-strain curve of sample B.

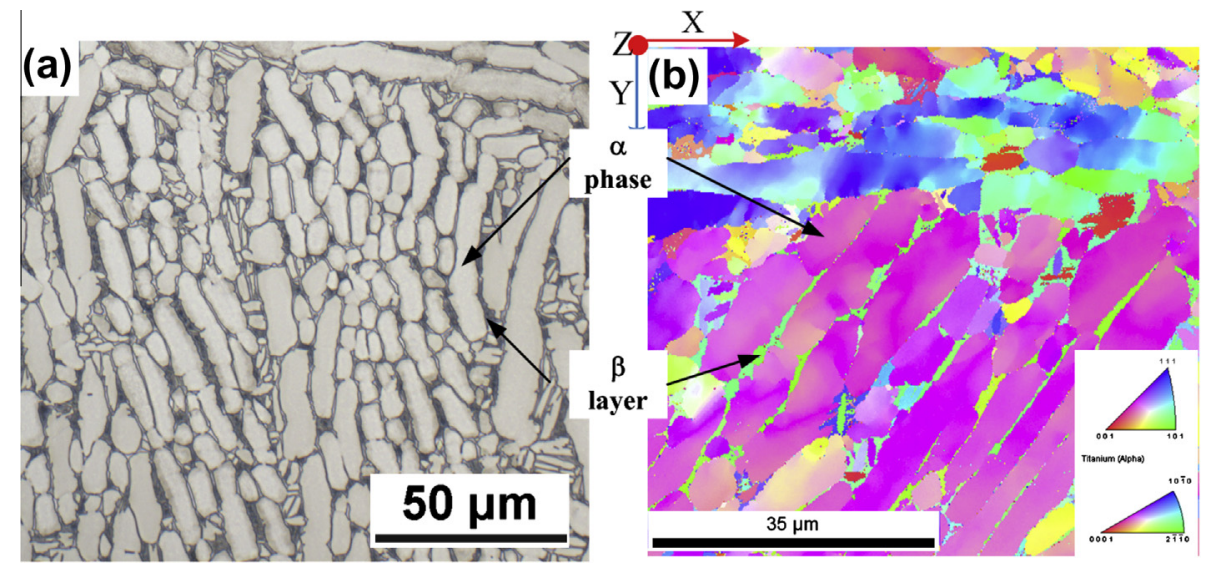

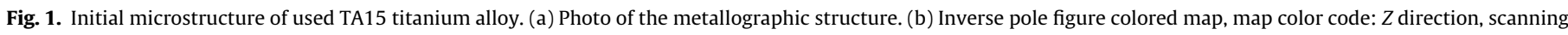
step size: $0.2 \mu \mathrm{m}$. 


\section{Results and discussion}

\subsection{Slip activation and transmission}

Fig. 3a shows an orientation map of sample A. The tensile direction is parallel to the $X$ direction. The microstructure of the mapped region consists of coarse $\alpha$ lamellae and thin $\beta$ layers. The volume fractions of the $\alpha$ and the $\beta$ phases (calculated by TSL/OIM analysis software) are $89 \%$ and $11 \%$, respectively. The $\alpha$ lamellae are $19 \mu \mathrm{m}$ in length and $5 \mu \mathrm{m}$ in thickness on average. Almost all of the $\alpha$ lamellae are parallel to each other and inclined at an angle of $\sim 45^{\circ}$ to the horizontal direction (tensile direction). Furthermore, all $\alpha$ lamellae exhibit a similar crystal orientation. The retained $\beta$ layers distributed between the $\alpha$ lamellae have a thickness in the range of $0.5-4 \mu \mathrm{m}$ (see Fig. 3a).

The corresponding image quality (IQ) map is shown in Fig. $3 \mathrm{~b}$. The slip traces are clearly observed in the $\alpha$ lamellae. In order to identify the activated slip system, all possible glide planes in the crystal coordinate system were calculated by the TSL/OIM software V5.31. The comparison between the observation and the calculated glide plane traces (dotted blue line) indicates that all of the observed glide planes correspond to the basal planes (0001). The active slip system is therefore $\{0001\}\langle 11-20\rangle$ basal slip.

The calculation results indicate a high Schmid factor (SF) of about $0.41-0.48$ for the basal $\{0001\}\langle 11-20\rangle$ slip system and a low value of about $0.21-0.28$ for the prismatic $\{10-10\}\langle 11-20\rangle$ slip system. Because the SF for the basal slip system is approximately two times larger than that for the prismatic slip system, basal slip must be dominant in the observed zone of $\alpha$ lamellae at early yielding (see Fig. $3 \mathrm{~b}$ ).

Another point concerns the slip transmission phenomenon at the grain/phase boundary. While some slip lines penetrate across the $\beta$ layers into the neighboring $\alpha$ grains as indicated by the red arrow at positions 1 and 2 in Fig. $3 \mathrm{~b}$; some other slip lines are end at the grain boundary, (the red arrow at position 3 in Fig. 3b). Actually, the conditions of grain a, b and c are very similar with respect to crystal orientation, strain/stress tensor and even the morphology of the $\alpha$ grains. The only difference between these positions concerns the crystal orientation relationship between the $\beta$ layer and its neighboring $\alpha$ grains.

The crystal orientations of the $\alpha$ lamellae and their $\beta$ neighbors at the positions 1,2 and 3 are represented by combined pole figures (PFs) of $\{110\}_{\beta} /\{0002\}_{\alpha}$ and $\{111\}_{\beta} /\{11-20\}_{\alpha}$ in Fig. $3 c$ $(\alpha$ : red and green dot, $\beta$ : blue square), respectively. These results indicate that the orientation relationship between the $\alpha$ lamellae and the neighboring $\beta$ layers have a significant influence on the slip transmission.

When the Burgers orientation relationship (OR) $\left(\{0002\}_{\alpha-}\right.$ $\left.\left\|\{110\}_{\beta},\langle 11-20\rangle_{\alpha}\right\|\langle 111\rangle_{\beta}\right)$ [20] is only partially maintained, where the $[-111]_{\beta}$ direction is almost parallel to $[-12-10]$ of $\alpha 1$ and $\alpha 2$ (only $4.0^{\circ} / 3.73^{\circ}$ and $4.2^{\circ} / 3.19^{\circ}$ misaligned respectively, as shown in Table 1$)$ but the $(-10-1)_{\beta}$ and $(0002)_{\alpha}$ planes are misaligned by approximately $10-20^{\circ}$ (all the misalignment angles between two planes are calculated by the plane normal vectors in the current presentation), as in the case for positions 1 and 2 (see first line in Fig. 3c), slip still can easily transfer from one $\alpha$ lamella into another neighboring $\alpha$ grain by activating the related slip system of $\beta$ layer, as shown in positions 1 and 2 of Fig. $3 \mathrm{~b}$. However, it is worth noting that the gray value of the slip line is lighter after transmission, especially for position 2 , where the two $\alpha$ grains (grain $\mathrm{b}$ and $\mathrm{c}$ ) are misaligned by relatively larger angles $\left(7.52^{\circ}\right.$ and $8.70^{\circ}$ for $(0002)_{\alpha}$ plane and $[-12-10] \alpha$ direction, respectively) comparing with the situation at position $1\left(2.76^{\circ}\right.$ and $\left.1.80^{\circ}\right)$. It is supposed that some dislocations piled-up at $\alpha / \beta$ interface [13], consequently, the slip in grain $b$ (seeing position 2 in Fig. 3b) has been somewhat suppressed.

When the neighboring grains are not Burgers OR (see line 3 in Fig. 3c), where both the $[-111]_{\beta} /[-12-10]_{\alpha}$ directions and the normal direction of the $(-10-1)_{\beta} /(0002)_{\alpha}$ planes are misaligned by the angle of $12-16^{\circ}$ and $13-19^{\circ}$ respectively (see Table 1 ), the slip lines in grain $c$ are stopped at the $\alpha / \beta$ interface and a completely new slip trace is observed in grain b, as shown in Fig. 3b position 3. Similar TEM observations on the influence of Burgers OR were made in [13,21-23]. However, the current observations are made in EBSD orientation maps which can deliver both slip line morphology and crystal orientation information. Furthermore, the situations at positions 1,2 and 3 in current presentation are fully comparable; so the results are relatively clear and easy to understand.

The slip transmission through $\beta$ layers into neighboring $\alpha$ grains has been described in some detail in literatures [14,24,25], and the mechanisms of slip transmission in single-colony crystals of an $\alpha / \beta$ titanium alloy have been discussed based on dislocation characterization [13]. However, as far as we know, the morphological
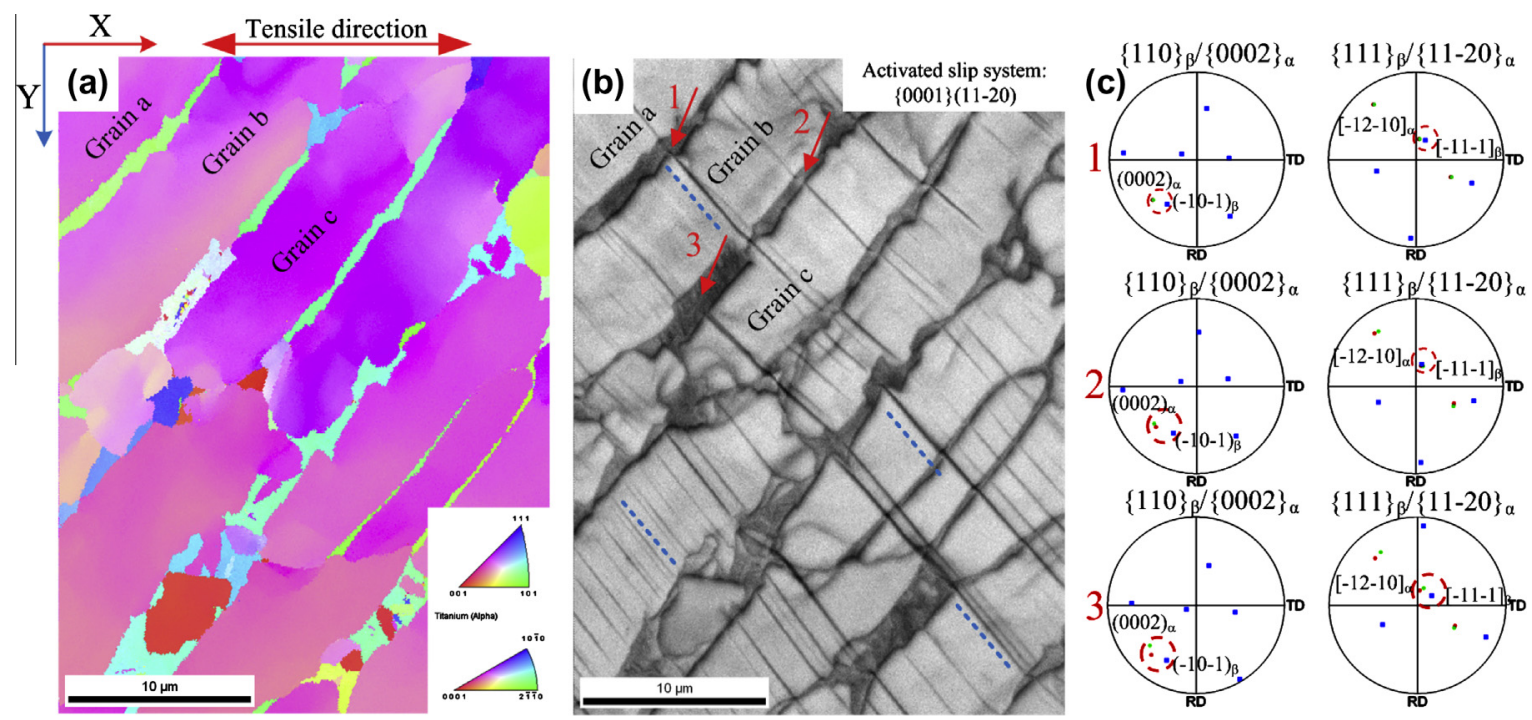

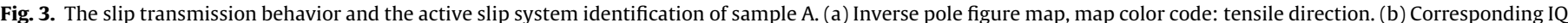

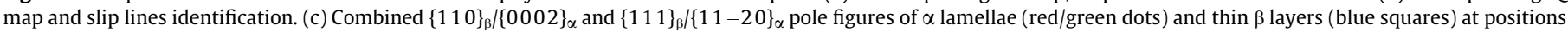
1,2 and 3. (For interpretation of the references to color in this figure legend, the reader is referred to the web version of this article.) 
Table 1

The misalignment angles between slip planes/slip directions at positions 1, 2 and 3 (Fig. 3b).

\begin{tabular}{|c|c|c|c|c|c|}
\hline Position & Slip plane or direction & $\alpha 1 / \alpha 2$ & Slip plane or direction & $\alpha 1 / \beta$ & $\alpha 2 / \beta$ \\
\hline Point 1 & $\begin{array}{l}(0002)_{\alpha 1} /(0002)_{\alpha 2} \\
{[-12-10]_{\alpha 1} /[-12-10]_{\alpha 2}}\end{array}$ & $\begin{array}{l}2.76^{\circ} \\
1.80^{\circ}\end{array}$ & $\begin{array}{l}(0002)_{\alpha} /(-10-1)_{\beta} \\
{[-12-10]_{\alpha} /[-111]_{\beta}}\end{array}$ & $\begin{array}{l}9.65^{\circ} \\
4.00^{\circ}\end{array}$ & $\begin{array}{r}12.40^{\circ} \\
3.73^{\circ}\end{array}$ \\
\hline Point 2 & $\begin{array}{l}(0002)_{\alpha 1} /(0002)_{\alpha 2} \\
{[-12-10]_{\alpha 1} /[-12-10]_{\alpha 2}}\end{array}$ & $\begin{array}{l}7.52^{\circ} \\
8.70^{\circ}\end{array}$ & $\begin{array}{l}(0002)_{\alpha} /(-10-1)_{\beta} \\
{[-12-10]_{\alpha} /[-111]_{\beta}}\end{array}$ & $\begin{array}{l}18.47^{\circ} \\
4.20^{\circ}\end{array}$ & $\begin{array}{r}20.63^{\circ} \\
3.19^{\circ}\end{array}$ \\
\hline Point 3 & $\begin{array}{l}(0002)_{\alpha 1} /(0002)_{\alpha 2} \\
{[-12-10]_{\alpha 1} /[-12-10]_{\alpha 2}}\end{array}$ & $\begin{array}{l}8.66^{\circ} \\
8.20^{\circ}\end{array}$ & $\begin{array}{l}(0002)_{\alpha} /(-10-1)_{\beta} \\
{[-12-10]_{\alpha} /[-111]_{\beta}}\end{array}$ & $\begin{array}{l}13.37^{\circ} \\
15.37^{\circ}\end{array}$ & $\begin{array}{l}18.15^{\circ} \\
12.35^{\circ}\end{array}$ \\
\hline
\end{tabular}

evolution of slip lines during transmission through the $\beta$ layer is described here for the first time.

\subsection{Slip line deflection}

To further reveal the slip line morphology evolution during transmission, another region on the sample A was investigated by SEM/EBSD. Fig. 4a presents the slip lines transferring across $\beta$ layer (b) into neighboring $\alpha$ grain (a1). As in the previous case, the slip systems in both $\alpha$ grains (a1 and a2) are identified as $\{0002\}\langle 11-20\rangle$ (see Fig. 4a). Some of the slip lines which transmit through the $\beta$ layer (b) keep straight morphology after crossing into the $\beta$ layer (b), as indicated by the white dotted line in Fig. 4a. On the other hand, some slip lines are deflected in the $\beta$ layer and retained the same direction in neighboring $\alpha$ grain as before transferring, as marked by the red, green, and blue dotted lines. Therefore, although the slip lines are continuous, various deflections are observed in the $\beta$ layer.

Fig. 4b shows a schematic explanation for the observation of the deflected slip lines. The blue lines which are plotted according to the slip lines in Fig. 4a, represent the traces of $\{0002\}$ slip planes in the $\alpha$ grains a1 and a2. The fine red, blue and black lines indicate the slip traces of all equivalent $\{110\},\{112\}$ and $\{123\}$ slip planes in the $\beta$ layer, respectively. The longer is a fine trace line, the more perpendicular does the respective plane stand with respect to the observation plane.

We must note that the Burgers OR is not well respected in this situation: misaligned angle between $(0002)_{\alpha}$ of grain a1/a2 and $(-10-1)_{\beta}$ of $\beta$ layer $b$ is approximately $20.15^{\circ}$, as shown in Fig. $4 \mathrm{c}$ and Table 2. Although the slip transmission must be increasingly difficult with the enhancement (or increase) of parallel misalignment [14], according to our previous experimental observations (see position 2 in Fig. 3) and discussions, slips still could transmit through the $\beta$ layer via $\{110\}_{\beta}$ slip system activation. However, $\{112\}$ and $\{123\}$ are also the possible slip systems in
Table 2

The misalignment angles between slip planes of $\alpha$ grain and $\beta$ layer.

\begin{tabular}{llll}
\hline Slip plane & $\begin{array}{l}(0002)_{\alpha} / \\
(-10-1)_{\beta}\end{array}$ & $\begin{array}{l}(0002)_{\alpha} / \\
(-112)_{\beta}\end{array}$ & $\begin{array}{l}(0002)_{\alpha} \\
(-12-3)_{\beta}\end{array}$ \\
\hline $\begin{array}{c}\text { Misaligned } \\
\text { angles }\end{array}$ & $20.15^{\circ}$ & $6.20^{\circ}$ & $1.33^{\circ}$ \\
M factor & 0.937 & 0.992 & 0.997 \\
\hline
\end{tabular}

BCC $\beta$ titanium having the same Burgers vector of the type $\langle 111\rangle$ [26]. Furthermore, it is obvious that they keep almost parallel relationships between the $(0002)_{\alpha}$ and $(-112)_{\beta} 6.02^{\circ}$ misalignment or $(-12-3)_{\beta} 1.33^{\circ}$ misalignment here, as dot red ellipse indicated region in Fig. 4c.

The factor $M(M=\cos \alpha \cdot \cos \beta$, where $\alpha$ is the angle between the Burgers vectors in the two crystal components and $\beta$ is the angle between the lines intersected with the interface of grain a and b active slip planes) are $0.937,0.992$ and 0.997 for $(0002)_{\alpha} /(-10-1)_{\beta}$, $(0002)_{\alpha} /(-112)_{\beta}$, and $(0002)_{\alpha} /(-12-3)_{\beta}$ respectively. According to the slip transmission criterion, slip is favored with maximum factor $M$ [14]. Actually, the $M$ values in the above-mentioned three cases are highest in their own equivalent slip system and close to each other, especially for the latter two cases. This means that the dislocations sliding in $(0002)_{\alpha}$ may also easily enter into either $(-112)_{\beta}$ or $(-12-3)_{\beta}$. Because various equivalent $\{110\}_{\beta},\{112\}_{\beta}$ or $\{123\}_{\beta}$ slip planes could be selected when slip transfers across $\beta$ layers, the slip traces exhibit straight or deflected morphology.

\subsection{Fork-like slip lines}

For sample B with $4.2 \%$ macroscopic deformation strain, differently magnified SEM images of the microstructure are presented in Fig. $5 a$ and b, respectively. As opposed to sample A, beside basal slip system (indicated by red dotted lines), activation of prismatic
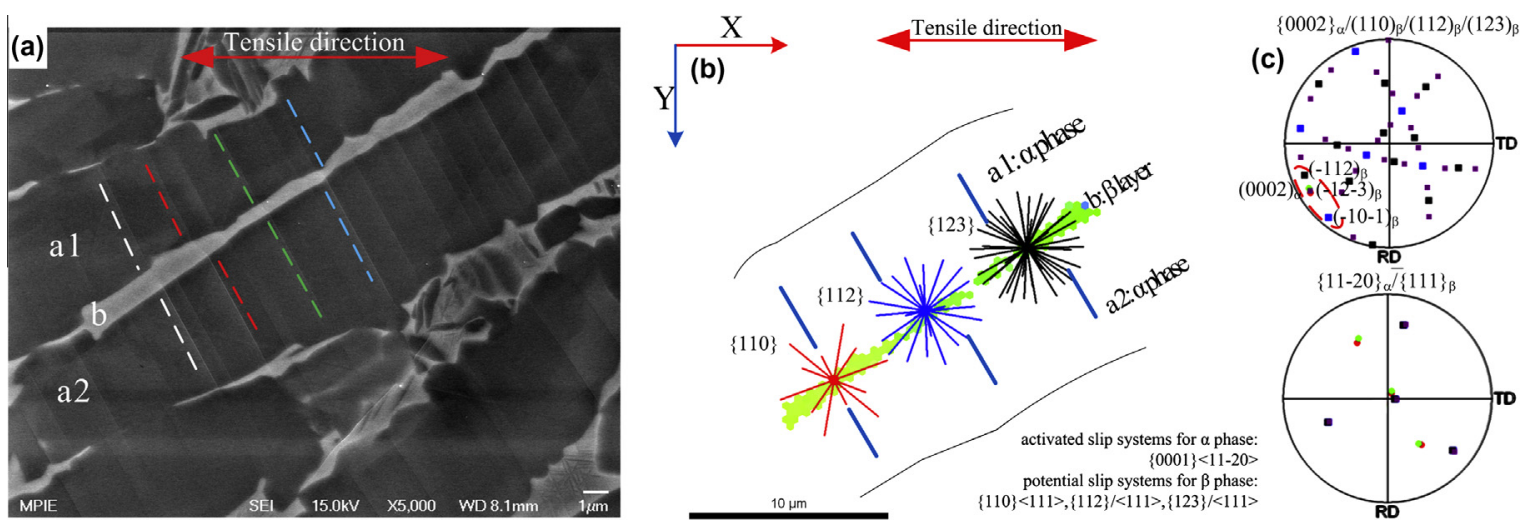

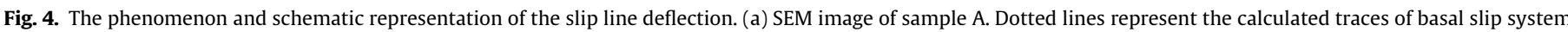

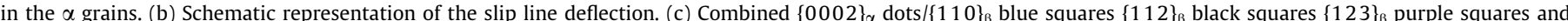

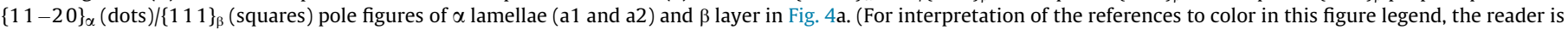
referred to the web version of this article.) 

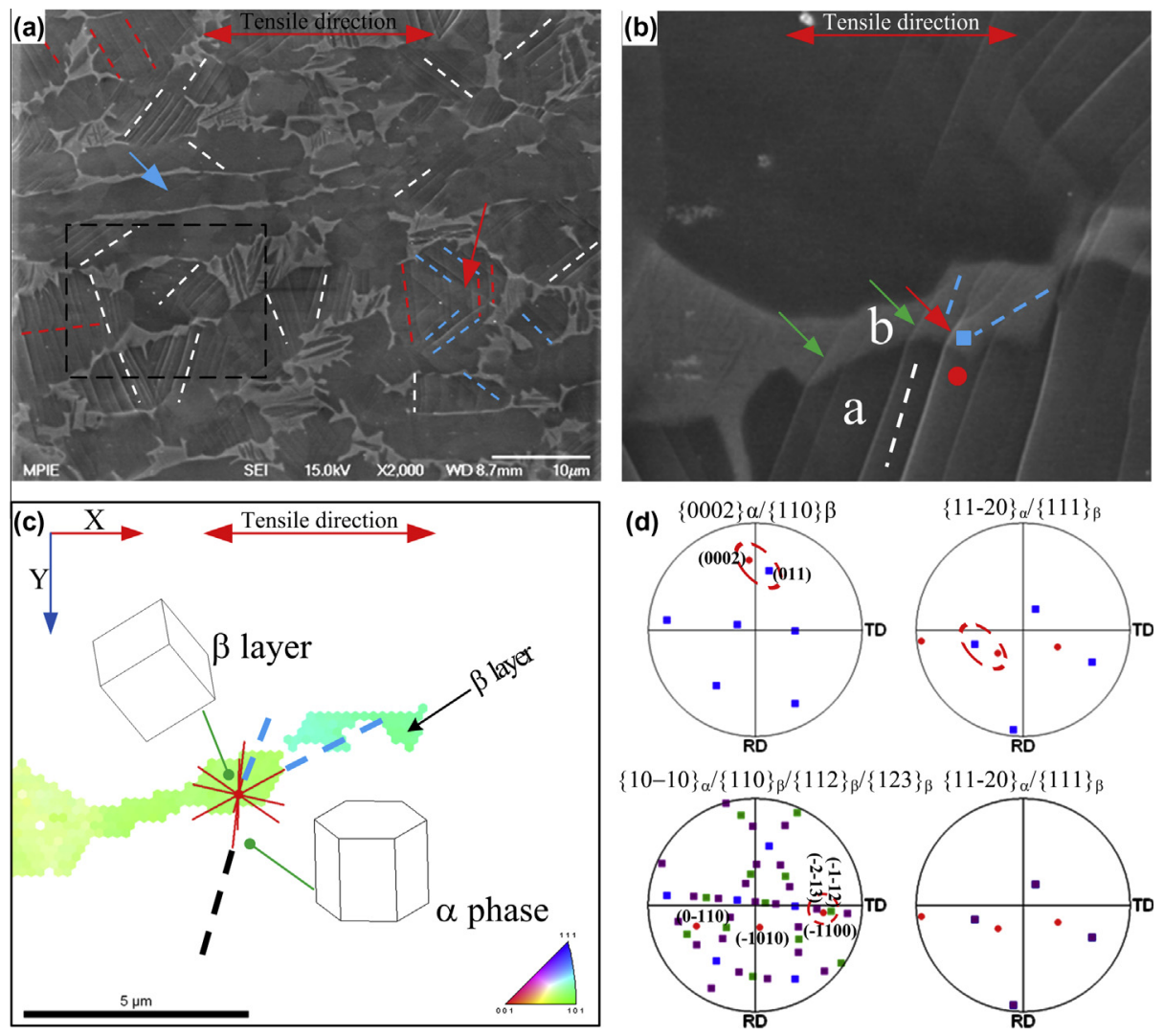

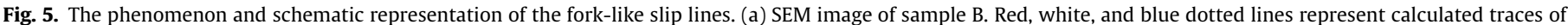

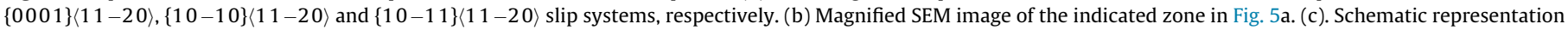

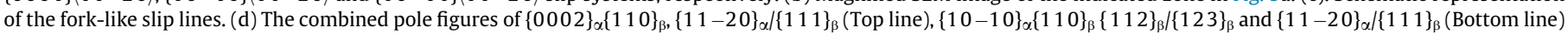

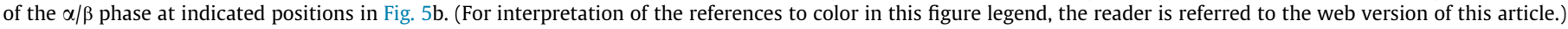

and $\langle a\rangle$-pyramidal slip systems is also detected, as indicated by the white and blue dotted lines in Fig. 5a. Obviously, the spatial distribution of activated slip systems is relatively inhomogeneous. For example, two coexisting slip systems are presented in the grain marked by a red arrow in Fig. 5a. No obvious slip lines are detected in the grains marked by the blue arrow (Fig. 5a), although the SF value is high (0.4-0.5) for prismatic slip system. Ankem and Margolin [27] have shown that the elastic compatibility stress between $\alpha$ and $\beta$ phases in lamellar colonies of titanium alloys can inhibit or favor some slip systems depending on the applied stress orientation. Castany et al. [28] also pointed out that the basal glide is slightly favored in lamellar colonies in Ti-6Al-4V alloys. This spatial heterogeneity indicates that, besides crystallographic orientation, other factors, such as grain morphology and neighboring grains, also influence the slip nature during plastic deformation.

Moving the focus to the morphology of slip lines, another novel phenomenon is observed which is marked by a red arrow in Fig. 5b. The consecutive slip line divides into two fine slip lines (looking like a "fork") when it transfers across the $\beta$ layer. Based on the corresponding EBSD data, the special slip lines are identified and the crystal frame of $\alpha$ and $\beta$ near the "fork" are presented, as shown in Fig. 5c.

Obviously, the Burgers OR is fully lost here (as shown the combined pole figure on the top of Fig. 3d), because the minimum misaligned angles between $\{0002\}_{\alpha}$ and $\{110\}_{\beta}$ or $\langle 11-20\rangle_{\alpha}$ and / $\langle 111\rangle_{\beta}$ are $\sim 20^{\circ}$ (see Table 3). According to our previous experi- mental results and other works, the slip transmission at the $\alpha / \beta$ interfaces should be difficult, if the Burgers OR is fully lost. Furthermore, the SFs of $\alpha$ nodule (marked a) for basal slip system are less than 0.1 , which means that there is no basal slips being activated in the marked grain a.

However, the slip transmissions are still detected and they exhibit a fork-like morphology in the $\beta$ layer. Here the activated slip system is identified to be $\{10-10\}\langle 11-20\rangle$ in grain a. One may ask whether the fork-like morphology results from activated cross slips on different prismatic planes? In fact, the SFs in grain a are $0.40,0.46$ and 0.06 for $(-1100)[-1-120],(-1010)[-12-10]$ and $(0-110)[2-1-10]$ slip system, respectively. Although it is hard to identify the activated slip systems, there is no doubt that the $(0-110)[2-1-10]$ slip systems are difficult to active here due to the low SF value. Even if both $(-1100)[-1-120]$ and $(-1010)[-12-10]$ slip systems have been activated, it still does not necessarily lead to the emergence of "fork" morphology. This is because that all potential slip planes of $\beta$ layer (marked "b", Fig. 5b) are severely misaligned with $(-1010)$ slip plane (bottom left of Fig. 5d, pole figure). Large misalignment angle between them means that it is very difficult for dislocation entering $\beta$ slip planes from ( -1010$)$ slip plane of $\alpha$. Therefore, "fork" morphology in the $\beta$ layer may not result from cross slip on different prismatic planes.

Another possible reason is that two slip systems could be activated during the slip transmitting through the $\beta$ layer. This means

Table 3

The misaligned angles between the slip planes/slip directions at positions a and b (Fig. 5b).

\begin{tabular}{|c|c|c|c|c|}
\hline Slip plane or direction & $(000-2)_{\alpha} /(011)_{\beta}$ & {$[-1-120]_{\alpha} /[-11-1]_{\beta}$} & $(-1100)_{\alpha} /(-1-12)_{\beta}$ & $(-1100)_{\alpha} /(-2-13)_{\beta}$ \\
\hline Misaligned angles & $17.26^{\circ}$ & $24.05^{\circ}$ & $3.98^{\circ}$ & $4.75^{\circ}$ \\
\hline
\end{tabular}


that dislocations could enter into two slip planes of $\beta$ phase during this process. Actually the activated slip plan $(-1100)_{\alpha}$ are almost parallel to both $(-1-12)_{\beta}$ and $(-2-13)_{\beta}$ potential slip planes (see red dotted circle in bottom left pole figure of Fig. 5d). The misaligned angles between them are $3.98^{\circ}$ and $4.75^{\circ}$, respectively, as listed in Table 3. This means that it is relatively easy for dislocation to enter into one or both of these two $\beta$ slip plane from $(-1010)$ slip plane of $\alpha$. However, the SFs for both $(-1-12)[111] 0.21$ and $(-2-13)[111](0.19)$ slip systems are too low for dislocations to be entered. Thus, as extension of slip in the $\beta$ layer, the slip is tend to occur on some relatively easy slip systems with a relative high SF value (such as 0.47 for $(2-11)[-1-11]$ and 0.40 for $(21-1)[-11-1]$ or 0.43 for $(-2-1-3)[11-1]$ and 0.49 for $(-312)[11-1])$ by cross slip mechanism. Then the slip trace in $\beta$ layer will be deflected and the fork-like morphology is presented.

Based on the above mentioned analysis, although the Burgers OR is missed, the slip still can transmit across the $\beta$ layer through activating dislocations to enter the $\beta$ phase slip plane which is almost parallel with the slip plane of the neighboring $\alpha$ phase. Obviously, thickness of $\beta$ layer also has a significant influence on the slip transmission nature. If the $\beta$ layer is relatively thin, slip transmission directly occurs on the special slip planes of $\beta$ which is closely parallel to the activated slip plane of neighboring $\alpha$ grain, although the selected slip systems of $\beta$ layer may do not have the highest SF. However, if the $\beta$ layer is relatively thick, the slip is tend to move to some more easy slip system from firstly selected slip plane (if SF is relatively low) during slip transmission through the $\beta$ layer. This is why "fork" morphology is only observed at relatively thick positions (see red arrow in Fig. 5b) and deflected slip line at other positions (green arrow in Fig. 5b).

\section{Conclusions}

In summary, the retained $\beta$ layers have a significant influence on the slip transmission feature in TA15 near alpha titanium alloys during tensile deformation at room temperature. The slip can easily transfer across the $\beta$ phase layer into the neighboring $\alpha$ lamella when the Burgers OR is fully or partially respected. Furthermore, the misalignment angles between the two $\alpha$ lamellae adjacent to the $\beta$ layer also influence the slip transmission feature: when the misalignment largely deviates from the Burgers OR, the slip lines are terminated at $\alpha / \beta$ interface boundary.

However, the Burgers OR does not seem to be the requirement for slip transmission at $\alpha / \beta$ phase boundaries in the $\alpha / \beta$ titanium alloys during plastic deformation at room temperature. Although the Burgers OR is missed, the slip can still transmit across the $\beta$ layer through firstly activating the dislocations easy entering slip plane and then move to some easier slip systems through cross slip mechanism.

These results indicate that the slip line morphologies during transferring are mainly determined by both the activated slip systems of $\beta$ phase and the crystal orientation relationship between $\alpha$ and $\beta$ at phase boundaries. These novel phenomena of slip line can give us a deep understanding of the slip transmission at $\alpha / \beta$ phase boundary in titanium alloys.

\section{Acknowledgements}

The author (He Dong) gratefully acknowledges scholarship support from the China Scholarship Council (CSC). The author (He Dong) also wishes to thank Dr. Li (Yujiao Li) and Dr. B. Ravi Kumar for their language helps.

\section{References}

[1] He D, Zhu JC, Wang Y, Liu Y. A study of dynamic recrystallization in Ta15 titanium alloy during hot deformation by cellular automata model. Int J Mod Phys B 2009;23:934-9.

[2] Sun ZC, Yang H. Microstructure and mechanical properties of TA15 titanium alloy under multi-step local loading forming. Mat Sci Eng A-Struct 2009;523:184-92.

[3] Fan X, Yang H, Gao P. Deformation behavior and microstructure evolution in multistage hot working of TA15 titanium alloy: on the role of recrystallization. J Mater Sci 2011;46:6018-28.

[4] Sieniawski J, Filip R, Ziaja W. The effect of microstructure on the mechanical properties of two-phase titanium alloys. Mater Des 1997;18:361-3.

[5] He D, Zhu JC, Lai ZH, Liu Y, Yang XW. An experimental study of deformation mechanism and microstructure evolution during hot deformation of Ti-6Al2Zr-1Mo-1V alloy. Mater Des 2013;46:38-48.

[6] Song WQ, Sun S, Zhu S, Wang G, Wang J, Dargusch MS. Compressive deformation behavior of a near-beta titanium alloy. Mater Des 2012;34:739-45.

[7] Fan XG, Yang H, Gao PF. Prediction of constitutive behavior and microstructure evolution in hot deformation of TA15 titanium alloy. Mater Des 2013;51:34-42.

[8] Quan G-z, Lv W-q, Mao Y-p, Zhang Y-w, Zhou J. Prediction of flow stress in a wide temperature range involving phase transformation for as-cast Ti-6Al2Zr-1Mo-1V alloy by artificial neural network. Mater Des 2013;50:51-61.

[9] Movchan BA, Lemkey FD. Strength, ductility and superplasticity of microcrystalline two-phase materials. Mater Des 1996:17:141-9.

[10] Bridier F, Villechaise P, Mendez J. Analysis of the different slip systems activated by tension in a alpha/beta titanium alloy in relation with local crystallographic orientation. Acta Mater 2005;53:555-67.

[11] Numakura H, Minonishi Y, Koiwa M. (1123)(1011) Slip in titanium polycrystals at room-temperature. Scripta Metall Mater 1986;20:1581-6.

[12] Bridier F, Villechaise P, Mendez J. Slip and fatigue crack formation processes in an alpha/beta titanium alloy in relation to crystallographic texture on different scales. Acta Mater 2008;56:3951-62.

[13] Suri S, Viswanathan GB, Neeraj T, Hou DH, Mills MJ. Room temperature deformation and mechanisms of slip transmission in oriented single-colony crystals of an alpha/beta titanium alloy. Acta Mater 1999;47:1019-34.

[14] Kehagias T, Komninou P, Dimitrakopulos GP, Antonopoulos JG, Karakostas T. Slip transfer across low-angle grain-boundaries of deformed titanium. Scripta Metall Mater 1995;33:1883-8.

[15] Li LX, Lou Y, Yang LB, Peng DS, Rao KP. Flow stress behavior and deformation characteristics of Ti-3Al-5V-5Mo compressed at elevated temperatures. Mater Des 2002;23:451-7.

[16] Gao PF, Yang H, Fan XG. Quantitative analysis of the microstructure of transitional region under multi-heat isothermal local loading forming of TA15 titanium alloy. Mater Des 2011;32:2012-20.

[17] Banerjee D, Williams JC. Microstructure and slip character in titanium alloys. Defence Sci J 1986;36:191-206.

[18] Donachie MJ. Titanium: a technical guide. 2nd ed. Metals Park, OH: ASM International; 1988.

[19] ISO 6892-1:2009, Metallic materials-tensile testing-Part 1: method of test at room temperature. International Organization for Standardization, 2009.

[20] Burgers WG. On the process of transition of the cubic-body-centered modification into the hexagonal-close-packed modification of zirconium. Physica 1934;1:561-86.

[21] Ankem S, Margolin H. Alpha-beta interface sliding in Ti-Mn alloys. Metall Mater Trans A 1983;14:500-3.

[22] Furuhara T, Ogawa T, Maki T. Atomic-structure of interphase boundary of an alpha-precipitate plate in a beta-Ti-Cr alloy. Phil Mag Lett 1995;72:175-83.

[23] Mills MJ, Hou DH, Suri S, et al. Orientation relationship and structure of alpha/ beta interfaces in conventional titanium alloys. In: Pond RC, Clark WAT, King $\mathrm{AH}$, et al., editors. Boundaries \& interfaces in materials. Warrendale: The Minerals, Metals and Materials Society (TMS); 1998. p. 295-301.

[24] Wiezorek JMK, Kulovits A, Zhang XD, Fraser HL. Slip transfer across heterointerfaces in two-phase titanium aluminum intermetallics. Metall Mater Trans A 2011;42A:605-12.

[25] Hu D, Loretto MH. Slip transfer between lamellae in fully lamellar TiAl alloys. Intermetallics 1999;7:1299-306.

[26] Lütjering G, Williams JC. Titanium. 2nd ed. New York: Springer, Berlin Heidelberg; 2007.

[27] Ankem S, Margolin H. The role of elastic interaction stresses on the onset of plastic flow for oriented two ductile phase structures. MTA 1980;11:963-72.

[28] Castany P, Pettinari-Sturmel F, Crestou J, Douin J, Coujou A. Experimental study of dislocation mobility in a Ti-6Al-4V alloy. Acta Mater 2007;55:6284-91. 